\title{
CORRIGENDUM
}

\section{Germline mutations predisposing to diffuse large B-cell lymphoma}

OC Leeksma, NF de Miranda and H Veelken

Blood Cancer Journal (2017) 7, e541; doi:10.1038/bcj.2017.22; published online 10 March 2017

Correction to: Blood Cancer Journal (2017) 7, e532; doi:10.1038/ bcj.2017.15; published online 17 February 2017
Following the online publication of this article, the author noticed an error in Table 1. Reference 3030 should be 29 as both LIG4 mutations originate from the same source. 